\title{
ON THE LOGIC OF FACTUAL EQUIVALENCE
}

\author{
FABRICE CORREIA \\ Université de Neuchâtel
}

\begin{abstract}
Say that two sentences are factually equivalent when they describe the same facts or situations, understood as worldly items, i.e. as bits of reality rather than as representations of reality. The notion of factual equivalence is certainly of central interest to philosophical semantics, but it plays a role in a much wider range of philosophical areas. What is the logic of factual equivalence? This paper attempts to give a partial answer to this question, by providing an answer the following, more specific question: Given a standard propositional language with negation, conjunction and disjunction as primitive operators, which sentences of the language should be taken to be factually equivalent by virtue of their logical form? The system for factual equivalence advocated in this paper is a proper fragment of the first-degree system for the logic of analytic equivalence put forward in the late seventies by R. B. Angell. I provide the system with two semantics, both formulated in terms of the notion of a situation's being fittingly described by a linguistic item. In the final part of the paper I argue, contra a view I defended in my "Grounding and Truth-Functions" (2010), that the logic for factual equivalence I advocate here should be preferred to Angell's logic if one wishes to follow the general conception of the relationships between factual equivalence and the notion of grounding put forward in the 2010 paper.
\end{abstract}

Say that two sentences are factually equivalent when they describe the same facts or situations, understood as worldly items, i.e. as bits of reality rather than as representations of reality. Factual equivalence is a form of "sameness of content", but it is to be distinguished from synonymy: the former is sameness of worldly content, whereas the latter is sameness of representational content. Synonymous sentences-at least, synonymous sentences which are in the business of stating facts-must be factually equivalent, but factually equivalent sentences need not be synonymous. Thus, for instance, 'Hesperus is Phosphorus' and 'Hesperus is Hesperus' are factually equivalent but not synonymous, and the same goes for 'The Mediterranean Sea contains water' and 'The Mediterranean Sea contains a substance composed of $\mathrm{H}_{2} \mathrm{O}$ molecules'.

The notion of factual equivalence is certainly of central interest to philosophical semantics. But the range of philosophical areas to which it is relevant is much broader. For talk of facts occurs in theories from all philosophical disciplines, and wherever this is so, the notion of factual equivalence has its place-if not de facto, at least de jure.

Under which conditions are two sentences factually equivalent? Part of an answer to this question is given by answering this other question: What is the logic of factual equivalence? This paper attempts to give a partial answer to this question, by addressing the following, narrower yet important question: Given a standard propositional language with negation, conjunction and disjunction as primitive operators, which sentences of the language should be taken to be factually equivalent by virtue of their logical form?

Received: December 30, 2014. 
An answer to this very question can be found in my "Grounding and Truth-Functions" (2010): two sentences $A$ and $B$ are factually equivalent by virtue of their logical form just in case $A$ and $B$ are provably equivalent according to the logic of analytic equivalence put forward by Angell $(1977,1989) .{ }^{1}$ I justified the proposal on the basis of considerations concerning the relation between factual equivalence and the notion of grounding (i.e. of a fact obtaining in virtue of other facts), but the justification I offered was only partial.

In this paper I put forward a logic for factual equivalence which is not Angell's logic but a proper fragment of it, and I attempt to fully justify my proposal. The justification I offer is semantical. Two semantics for the logic are in fact given, both formulated in terms of the notion of a situation's being fittingly described by a linguistic item. One of the semantics is a variant of a "dual" version of the semantics for Angell's logic I introduced in Correia (2004), and the other one is a "state space" semantics à la Kit Fine (see Fine, 2012a; 2012b; 2014; forthcoming; ms a; ms b; ms c; Fine's forthcoming paper is especially relevant). A key (and somewhat surprising) feature of the logic I advocate is that, unlike Angell's logic, it does not validate the distributivity principle according to which $A \vee(B \wedge C)$ is always equivalent to $(A \vee B) \wedge(A \vee C)$. In the final part of the paper, I argue that if one wishes to endorse the approach to the relation between grounding and factual equivalence I advocated in the 2010 article, one should prefer the logic for factual equivalence I advocate in the present paper to Angell's logic. ${ }^{2}$

§1. Situations and their descriptions. The core semantic idea which drives my theorising about factual equivalence is that

(FE) Two sentences $A$ and $B$ are factually equivalent just in case the situations described by $A$ are the same as the situations described by $B .^{3}$

Notice that in (FE), the term 'situation' rather than 'fact' is used to pick out the worldly content of sentences. The reason is that 'fact' is most often used in such a way that all the relevant instances of 'if $A$ describes a fact, then $A$ is true' are true, while on my conception of worldly content, whether a sentence has a nonempty worldly content does not turn on whether it is true. Also notice that I used 'situations' rather than 'situation' in the formulation of (FE): I will indeed take it that some sentences describe more than one situation. An important class of examples is given by disjunctions. Thus, for instance, the sentence 'Brutus is a dog or Maria is upset' will describe situations which are described by 'Brutus is a dog' but not by 'Maria is upset', as well as situations which are described by 'Maria is upset' but not by 'Brutus is a dog'.

Following a broadly Tractarian tradition, we may take a situation to consist in the obtaining of certain atomic states of affairs and the nonobtaining of certain atomic states

1 As I emphasised in the paper, the view is not that factual equivalence is a form of analytic equivalence (the opposite view would indeed fly in the face of the foregoing considerations about the distinction between factual equivalence and synonymy), but only that the two notions share certain logical features.

2 The semantic studies on Angell's logic are very few. Apart form the already cited Correia (2004) and Fine (forthcoming), Ferguson (2014) is worth mentioning.

3 I should stress that I take this account of factual equivalence to be compatible with the view that factual equivalence should ultimately be expressed by means of a sentential operator rather than by means of a predicate, and that the truth-conditions of statements of factual equivalence in operator form do not require an ontology of situations. 
of affairs. ${ }^{4}$ On that view, if $S$ and $T$ are two sets of atomic states of affairs, we may represent the situation consisting in the obtaining of the members of $S$ and the nonobtaining of the members of $T$ by means of the ordered pair $\langle T, S\rangle$. A situation may be possible or impossible, and complete (i.e. a world) or partial (i.e. a part of a world). Let $s$ be a situation represented by the pair $\langle T, S\rangle$. Then $s$ is impossible iff it is not possible, and possible iff it is possible that all the members of $S$ obtain and all the members of $T$ fail to obtain; and $s$ is partial iff it is not complete, and complete iff every atomic state of affairs belongs to $T \cup S$. (Notice that $s$ will be impossible if $T \cap S \neq \varnothing$, a case which is not excluded on the proposed conception of situations.) A situation may be part of another situation: the situation represented by $\langle T, S\rangle$ is part of the situation represented by $\left\langle T^{\prime}, S^{\prime}\right\rangle$ iff both $S \subseteq S^{\prime}$ and $T \subseteq T^{\prime}$. Situations also fuse: if $\left\langle T_{1}, S_{1}\right\rangle,\left\langle T_{2}, S_{2}\right\rangle, \ldots$ represent situations, then these situations have a fusion, represented by $\left\langle T_{1} \cup T_{2} \cup \ldots, S_{1} \cup S_{2} \cup \ldots\right\rangle$. Such a conception of situations, taken as a whole, is certainly not compulsory for our purposes, but taking it on board will be useful to guide our thoughts.

Consider, then, a given propositional language with negation, conjunction and disjunction as primitive operators. How can we describe situations using such a language? Two options naturally come to mind.

The first option is to use sentences of the language and to work with the semantic notion of a sentence describing a situation. On that view, it is then natural to distinguish between fitting and truncated descriptions. A truncated description of a situation, unlike a fitting description, ignores parts of the situation. As an illustration, consider the situation $s_{0}$ represented by

- 〈\{Sam's being French $\}$, \{Brutus’ being a dog, Maria’s being upset $\}\rangle$

Then the sentence

(a) 'Sam is not French and (Brutus is a dog and Maria is upset)'

is a fitting description of $s_{0}$, while the sentence

(b) 'Brutus is a dog'

describes it only in a truncated way.

The second option is somewhat more straightforward given the nature of the situations. The idea is that instead of working with the notion of a sentence describing a situation, one should work with the notion of a supersentence describing a situation. Syntactically, a supersentence can be taken to be a pair $L \mid R$, where $L$ and $R$ are collections of sentences

4 Some will take the idea of "the nonobtaining of atomic states of affairs" to be obscure or even unintelligible. But there are alternative approaches which make do without this idea. On one such approach, a situation consists in a bunch of states of affairs whose components are either atomic (Sam's being sad) or negations of atomic states of affairs (its not being the case that Sam is sad). This approach, too, might be taken to be problematic: negation operates on representations, one might object, not on worldly items such as states of affairs. Another alternative approach, which escapes this objection, is to follow Barker \& Jago (2012) on atomic states of affairs. On their view, we must countenance a relation of anti-instantiation alongside the familiar relation of instantiation. An atomic state of affairs may then be either positive, i.e. consisting in some object(s) instantiating an attribute (e.g. Sam's being sad, which consists in Sam's instantiating sadness), or negative, i.e. consisting in some object(s) anti-instantiating an attribute (e.g. Sam's not being sad, which consists in Sam's anti-instantiating sadness). On the approach under consideration, a situation consists in a bunch of positive and / or negative atomic states of affairs. 
such that $L \cup R \neq \varnothing$. (I use $L \mid R$ rather than $\langle L, R\rangle$ for reasons of convenience.) Semantically, a supersentence $L \mid R$ is taken to "say" that all the members of $L$ are false and all the members of $R$ are true.

The distinction between fitting and truncated descriptions is also meaningful in this alternative framework. Thus, the situation $s_{0}$ introduced above is fittingly described by the supersentence

(c) $\{$ 'Sam is French' $\} \mid\{$ 'Brutus is a dog', 'Maria is upset' $\}$,

but is only described in a truncated way by the supersentence

(d) $\varnothing \mid\{$ 'Brutus is a dog' $\}$

and by the supersentence

(e) $\{$ 'Sam is French' $\} \mid$ \{'Brutus is a dog' $\}$.

In this framework, of course, description by single sentences can be seen as a special case of description by supersentences, since a sentence $S$ is a (fitting / truncated) description of a situation iff the supersentence $\varnothing \mid\{S\}$ is a (fitting / truncated) description of that situation.

Descriptions, be they fitting or truncated, can be more or less determinate or specific. Thus, both (a) and (c) are fitting descriptions of $s_{0}$ which are equally determinate - they are indeed both fully determinate. In contrast,

(f) 'Sam is not French and (Brutus is an animal and Maria is upset)' and

(g) $\{$ 'Sam is French' $\} \mid$ \{'Brutus is an animal', 'Maria is upset' $\}$

are still fitting (no part of the situation is left aside), equally determinate, but they are less determinate than (a) and (c). Or again, the sentence

(h) 'Brutus is an animal'

is a truncated description of $s_{0}$ which is less determinate than (b), and the supersentence

(i) $\{$ 'Sam is French' $\} \mid$ \{'Brutus is an animal' $\}$.

is a truncated description of the situation which is less determinate than (e).

Moving from a more determinate to a less determinate description involves a loss of information about the situation described, and the same goes when we move from fitting to truncated descriptions or from less truncated to more truncated descriptions. Yet it is of crucial importance to appreciate that the loss of information is of a different kind in each case: in the second case, part of the description is simply removed, whereas in the first case part of the description is replaced by something more generic.

Which conception of descriptions should be used if factual equivalence is to be characterised along the lines of (FE)? Irrespective of whether we opt for the sentential or the supersentential approach, there are four options one can naturally think of:

1. The relevant notion is that of a fitting description, with no constraint regarding specificity;

2. The relevant notion is that of a fitting-or-truncated description, with no constraint regarding specificity;

3. The relevant notion is that of a fitting, fully specific description;

4. The relevant notion is that of a fitting-or-truncated, fully specific description. 
Suggestions 3 and 4 are nonstarters, since disjunctions do not describe in a specific way except in special cases (e.g. the cases in which the two disjuncts of a disjunction are one and the same sentence, which specifically describes a situation). The option I had in mind when formulating (FE) was 1, and it is this option I will take on board for most of the remaining parts of this paper. I shall say a word about option 2 in the last two sections, showing that the resulting logic of factual equivalence is a well-known logic, and arguing that option 1 is best suited for the task of theorising about grounding along the lines suggested in Correia (2010).

In what follows I shall pursue the two semantical approaches spelt out above, the sentential approach and the supersentential approach.

The sentential semantics introduced in the next section is a "state space" semantics of the sort put forward by Kit Fine in a series of recent works (see all the papers by Fine in the bibliography), in particular in his own work on Angell's logic (Fine, forthcoming). It should be pointed out that Fine does not work with the notion of a sentence fittingly describing a situation, but rather with the notion of a situation (or fact, or state) exactly verifying a sentence, where a state exactly verifies a sentence when it verifies it and is "wholly relevant" to the truth of the sentence (Fine, 2012b; 2014; ms a; ms c). Yet I take the notion of being fittingly described by to provide a concrete specification of the somewhat underspecified notion of exactly verifying: a situation is taken to be "wholly relevant" to the truth of a sentence when the sentence describes all the aspects of the situation. ${ }^{5}$

The supersentential semantics, also introduced in the next section, is in a sense "dual" to the semantics for Angell's logic of analytic equivalence put forward in Correia (2004): in that paper, I also invoke supersentences, but they are interpreted disjunctively rather than conjunctively, and likewise the underlying conception of situations takes them to be disjunctive rather than conjunctive in nature.

\$2. Formal semantics. Our object language has two kinds of sentences, the truthfunctional sentences and the equivalences. The former are built from a collection of atomic sentences using negation $(\neg)$, conjunction $(\wedge)$ and disjunction $(\vee)$, and the latter have the form $A \approx B$, where $A$ and $B$ are truth-functional sentences. The symbol $\approx$ is used for factual equivalence. I shall reserve the term 'sentence' for truth-functional sentences and use exclusively 'equivalence' for expressions of type $A \approx B$.

Following standard custom I define a literal as a sentence which is either an atom or a negated atom, and in accordance with the considerations from the previous section I define a supersentence as a pair $L \mid R$, where $L$ and $R$ are sets of sentences such that $L \cup R \neq \varnothing$, requiring in addition that both $L$ and $R$ be finite. (In what follows, I shall use $L, R$ and variants thereof for sets of sentences, reserving $A, B, C, D$ and variants thereof for sentences.) A supersentence is said to be basic if the sentences which compose it are all atomic. Following standard notational conventions I will often write $L, L^{\prime} \mid R, R^{\prime}$ instead of $L \cup L^{\prime}\left|R \cup R^{\prime}, L\right| R, A$ instead of $L|R \cup\{A\}, L| A$ instead of $L \mid\{A\}$, etc.

Let us start with the supersentential semantics. We define a supermodel as a pair $\langle S, \Vdash\rangle$, where

5 An ancestor of Fine's semantics is the semantics introduced by van Fraassen (1969), by means of which he characterises the logic of first-degree entailments à la Anderson \& Belnap (1962, 1963). The state space semantic framework is very fruitful, as Fine's own work illustrates (see also Cobreros et al., 2014 for a further application). A referee for this journal pointed out to me that a similar kind of semantic approach has recently been independently pursued by Jennings \& Chen (2013). 
- $\quad S$ is a nonempty set, and

- $\quad \Vdash$ is a binary relation between situations and basic supersentences.

In such a structure, $S$ is thought of as a set of situations, and on the intended interpretation, if $s \in S$ and $L \mid R$ is a basic supersentence, to say that $s \Vdash L \mid R$ is to say that $L \mid R$ is a fitting description of $s$. Notice that it is not required that for every atom $p$, either $\varnothing \mid p$ or $p \mid \varnothing$ describes a situation, or that there is no atom $p$ such that both $\varnothing \mid p$ and $p \mid \varnothing$ describe some situations (it is indeed allowed that for $p$ atomic, some situation be described by both $\varnothing \mid p$ and $p \mid \varnothing)$.

Given a supermodel $\langle S, \Vdash\rangle$, the description relation $\Vdash$ is then extended to all the supersentences of the language thanks to the following recursive specification:

$$
\begin{array}{lr}
\text { 1. } s \Vdash L \mid R, \neg A \text { iff } s \Vdash L, A \mid R & (\text { where } \neg A \notin R) \\
\text { 2. } s \Vdash L, \neg A \mid R \text { iff } s \Vdash L \mid R, A & (\text { where } \neg A \notin L) \\
\text { 3. } s \Vdash L \mid R, A \wedge B \text { iff } s \Vdash L \mid R, A, B & (\text { where } A \wedge B \notin R) \\
\text { 4. } s \Vdash L, A \wedge B \mid R \text { iff } s \Vdash L, A \mid R \text { or } s \Vdash L, B \mid R \text { or } s \Vdash L, & A, B \mid R \\
& \text { (where } A \wedge B \notin L) \\
\text { 5. } s \Vdash L \mid R, A \vee B \text { iff } s \Vdash L \mid R, A \text { or } s \Vdash L \mid R, B \text { or } s \Vdash L \mid R, A, B \\
& \text { (where } A \vee B \notin R \text { ) } \\
\text { 6. } s \Vdash L, A \vee B \mid R \text { iff } s \Vdash L, A, B \mid R & \text { (where } A \vee B \notin L)
\end{array}
$$

On the intended interpretation, the extended relation $\Vdash$ is still taken to be the relation of being fittingly described by. With this in mind, the proposed Clauses 1-6 are-I take itutterly natural, if not compulsory. Notice that Clauses 4 and 5 would be problematic if $\Vdash$ were understood as the relation of being fittingly and fully determinately described by: if, say, $\varnothing \mid$ 'Brutus is a dog' is a fitting and fully determinate description of a situation, then $\varnothing \mid$ 'Brutus is a dog or Maria is upset' is still a fitting description, but it is not fully determinate. ${ }^{6}$

A simple proof, which is left to the reader, shows that the bracketed conditions in Clauses 1-6 can be dropped without affecting the truth of the biconditionals.

Given a supermodel $M=\langle S, \Vdash\rangle$, we adopt the following definitions:

- $A$ describes $s$ in $M$ iff $s \Vdash \varnothing \mid A$;

- $A \approx B$ holds in $M$ iff $A$ and $B$ describe the same situations in $M$.

And we put:

- $A \approx B$ is supervalid iff $A \approx B$ holds in every supermodel.

Let us now turn to the sentential (Finean) semantics. We define a model as a quadruple $\langle S, \sqcup, F, V\rangle$, where

- $S$ is a nonempty set,

${ }^{6}$ Clause 5 may strike one as less natural than the following clause:

$$
5^{*} . s \Vdash L \mid R, A \vee B \text { iff } s \Vdash L \mid R, A \text { or } s \Vdash L \mid R, B
$$

(where $A \vee B \notin R$ )

But the conception of descriptions as fitting requires the more complex clause: if $A$ and $B$ together constitute a fitting description of a situation, then so does $A \vee B$ (even though in many cases it will be less specific than the description constituted by $A$ and $B$ taken together). A similar remark applies to clause 4 . 
- $\square$ an operation taking any nonempty subset of $S$ into a member of $S$, such that (i) for any $s \in S, \bigsqcup\{s\}=s$, and (ii) for any family $\left(S_{i}\right)_{i \in I}$ of nonempty subsets of $S$, $\bigsqcup\left\{\bigsqcup S_{i}: i \in I\right\}=\bigsqcup \bigcup\left\{S_{i}: i \in I\right\}$, and

- $F$ (falsification) and $V$ (verification) are partial functions from atoms to nonempty subsets of $S$, such that: (i) for any atom $p$ such that $F(p) \neq \varnothing$ and any nonempty subset $T$ of $F(p), \sqcup T \in F(p)$ and (ii) for any atom $p$ such that $T(p) \neq \varnothing$ and any nonempty subset $T$ of $V(p), \sqcup T \in V(p)$.

In such a structure, $S$ is thought of as a set of situations, if $T$ is a nonempty subset of $S$, $\bigsqcup T$ is thought of as the fusion of the members of $T$, and if $p$ is an atom, $F(p)$ is thought of as the set of situations that falsify $p$ and $V(p)$ as the set of situations that verify it. Notice that it is not required that every atom has a falsifier or a verifier, or that no atom has both a verifier and a falsifier (an atom is indeed allowed to be both verified and falsified by the same situation).

Given a model $\langle S, \sqcup, F, V\rangle$, the verification relation $\vDash$ and the falsification relation $=$ are then defined as follows:

1. $s \vDash p$ iff $s \in V(p)$

2. $s=p$ iff $s \in F(p)$

3. $s \vDash \neg A$ iff $s \neq A$

4. $s \exists \neg A$ iff $s \vDash A$

5. $s \vDash A \wedge B$ iff for some $t, u \in S$ with $s=t \bigsqcup u, t \vDash A$ and $u \vDash B$

6. $s=A \wedge B$ iff $s \Rightarrow A$ or $s=B$ or for some $t, u \in S$ with $s=t \bigsqcup u, t \exists A$ and $u \neq B$

7. $s \vDash A \vee B$ iff $s \vDash A$ or $s \vDash B$ or for some $t, u \in S$ with $s=t \bigsqcup u, t \vDash A$ and $u \vDash B$

8. $s=A \vee B$ iff for some $t, u \in S$ with $s=t \bigsqcup u, t=A$ and $u=B$

(I use $t \bigsqcup u$ for $\bigsqcup\{t, u\}$.) In our framework, a situation verifies a sentence iff the sentence is a fitting description of the situation, and a situation falsifies a sentence iff the negation of the sentence fittingly describes the situation (see the end of Section 1$)^{7}$

A proof by induction on the length of the sentences establishes that the closure condition imposed on the falsifiers and the verifiers of the atoms carries over arbitrary sentences: for any nonempty subset $T$ of falsifiers of $A, \bigsqcup T$ falsifies $A$, and for any nonempty subset $T$ of verifiers of $A, \bigsqcup T$ verifies $A$.

Given a model $N$, we say that

- $A \approx B$ holds in $N$ iff $A$ and $B$ have the same verifiers in $N$,

and we put:

- $A \approx B$ is valid iff $A \approx B$ holds in every model.

7 Why not use the familiar-sounding clause

7*. $s \vDash A \vee B$ iff $s \vDash A$ or $s \vDash B$

rather than than Clause 7? (Note that van Fraassen, 1969 does just this; Fine works with both clauses, depending on the target application (see Fine, ms c).) But here as in the case of the supersentential semantics, the conception of descriptions as fitting requires the more complex clause: if $A$ and $B$ constitute fitting descriptions of, respectively, $s_{1}$ and $s_{2}$, then $s_{1} \sqcup s_{2}$ will be fittingly described by $A \wedge B$, but also by $A \vee B$ (albeit in many cases less specifically). A similar remark applies to clause 6 . 
The sentential semantics exploits the mereological structure of situations, while the supersentential semantics does not. Yet given the adequacy results to be established in Section 5, supervalidity and validity are coextensive. The lack of structure on the "worldly side" of the semantic relation in the supersentential semantics is thus compensated by the extra structure on the "linguistic side" of the relation.

It is of course possible to devise a supersentential semantics which also exploits the mereological structure of situations. Such a semantics would actually collapse into the sentential semantics, since a supersentence $L \mid R$ (fittingly) describes a situation $s$ iff (i) each sentence in $L$ is (exactly) falsified by some parts of $s$, (ii) each sentence in $R$ is (exactly) verified by some parts of $s$, and (iii) $s$ is the fusion of all the parts which falsify members of $L$ or verify members of $R$. Yet a nice feature of the supersentential semantics as formulated above is that it provides a straightforward decision method for determining whether an equivalence is valid.

Assume given an enumeration of all the sentences, and another enumeration of all the supersentences. Where $\mathcal{S}$ is a finite nonempty set of supersentences, we define its reduction $r(\mathcal{S})$ as follows:

- If $\mathcal{S}$ only contains basic supersentences, $r(\mathcal{S})=\mathcal{S}$.

- If $\mathcal{S}$ contains some nonbasic supersentences, let $L \mid R$ be the first supersentence (in the given enumeration of the supersentences) in $\mathcal{S}$ that is nonbasic, and $A$ the first sentence (in the given enumeration of the sentences) in $L \mid R$ that is nonatomic. Then:

- In case $A=\neg B$ :

* If $L|R=L| R^{\prime}, A$ with $A \notin R^{\prime}$, then $r(\mathcal{S})$ results from $\mathcal{S}$ by removing $L \mid R$ and then adding $L, B \mid R^{\prime}$

* If $L\left|R=L^{\prime}, A\right| R$ with $A \notin L^{\prime}$, then $r(\mathcal{S})$ results from $\mathcal{S}$ by removing $L \mid R$ and then adding $L^{\prime} \mid R, B$

- In case $A=B \wedge C$ :

* If $L|R=L| R^{\prime}, A$ with $A \notin R^{\prime}$, then $r(\mathcal{S})$ results from $\mathcal{S}$ by removing $L \mid R$ and then adding $L \mid R^{\prime}, B, C$

* If $L\left|R=L^{\prime}, A\right| R$ with $A \notin L^{\prime}$, then $r(\mathcal{S})$ results from $\mathcal{S}$ by removing $L \mid R$ and then adding $L^{\prime}, B\left|R, L^{\prime}, C\right| R$ and $L^{\prime}, B, C \mid R$

- In case $A=B \vee C$ :

* If $L|R=L| R^{\prime}, A$ with $A \notin R^{\prime}$, then $r(\mathcal{S})$ results from $\mathcal{S}$ by removing $L \mid R$ and then adding $L\left|R^{\prime}, B, L\right| R^{\prime}, C$ and $L \mid R^{\prime}, B, C$

* If $L\left|R=L^{\prime}, A\right| R$ with $A \notin L^{\prime}$, then $r(\mathcal{S})$ results from $\mathcal{S}$ by removing $L \mid R$ and then adding $L^{\prime}, B, C \mid R$

Iterating the reduction operation on any finite set $\mathcal{S}$ of supersentences yields, after a finite number of steps, a set of basic supersentences we call $\mathcal{S}$ 's ultimate reduction.

Where $A$ is a sentence, let $|A|$ be the ultimate reduction of $\{\varnothing \mid A\}$. Clearly, for all supermodels $\langle S, \Vdash\rangle$ and all situations $s \in S, A$ describes $s$ iff for some $L \mid R$ in $|A|$, $s \Vdash L \mid R$. We then have:

LEMma 2.1. For all sentences $A$ and $B, A \approx B$ is supervalid iff $|A|=|B|$. 
Proof. The right-to-left direction is straightforward. For the other direction, suppose for illustration that there is a basic supersentence $L \mid R$ in $|A|$ not in $|B|$. Consider then a model $\langle\{s\}, \Vdash\rangle$ where $s \Vdash L^{\prime} \mid R^{\prime}$ iff both $L=L^{\prime}$ and $R=R^{\prime}$ for every basic supersentence $L^{\prime} \mid R^{\prime}$. Then $s$ is described by $A$ but not by $B$.

§3. The system. A system which is adequate with respect to both semantics is given by the following Hilbert-style axiomatisation:

\section{Axioms}

A1. $A \approx \neg \neg A$

A2. $A \approx A \wedge A$

A3. $A \wedge B \approx B \wedge A$

A4. $A \wedge(B \wedge C) \approx(A \wedge B) \wedge C$

A5. $A \approx A \vee A$

A6. $A \vee B \approx B \vee A$

A7. $A \vee(B \vee C) \approx(A \vee B) \vee C$

A8. $\neg(A \wedge B) \approx \neg A \vee \neg B$

A9. $\neg(A \vee B) \approx \neg A \wedge \neg B$

A10. $A \wedge(B \vee C) \approx(A \wedge B) \vee(A \wedge C)$

\section{Rules}

R1. $A \approx B / B \approx A$

R2. $A \approx B, B \approx C / A \approx C$

R3. $A \approx B / A \wedge C \approx B \wedge C$

R4. $A \approx B / A \vee C \approx B \vee C$

Adding the axiom dual to A10, namely

A11. $A \vee(B \wedge C) \approx(A \vee B) \wedge(A \vee C)$

yields R. B. Angell's first-degree system for analytic equivalence (see Fine, forthcoming). Interestingly, as Fine (forthcoming) has shown, A11 is equivalent to the rule

N. $A \approx B / \neg A \approx \neg B$

in the presence of axioms A1-A10 and rules R1-R4.

It may strike one as odd that A11 should not be countenanced. But given the foregoing semantic considerations, it is easy to see that $A \vee(B \wedge C)$ and $(A \vee B) \wedge(A \vee C)$ need not fittingly describe the same situations, and hence that A11 is not valid.

For consider the situations $s_{1}, s_{2}$ and their fusion $s_{3}$, whose descriptions are, respectively

1. $\langle\varnothing,\{$ Sam's being sad $\}\rangle$;

2. $\langle\varnothing,\{$ Sam's being ill $\}\rangle$;

3. $\langle\varnothing,\{$ Sam's being sad, Sam's being ill $\}\rangle$.

Then

- $\varnothing \mid$ 'Sam is sad' fittingly describes $s_{1}$

and hence

- $\varnothing \mid$ 'Sam is sad or Sam is bad' fittingly describes $s_{1}$. 
Also,

- $\varnothing \mid$ 'Sam is ill' fittingly describes $s_{2}$

and hence

- $\varnothing \mid$ 'Sam is sad or Sam is ill' fittingly describes $s_{2}$.

It follows that

- $\varnothing \mid$ '(Sam is sad or Sam is bad) and (Sam is sad or Sam is ill)' fittingly describes $s_{3}$.

But

- $\varnothing \mid$ 'Sam is sad or (Sam is bad and Sam is ill)' does not fittingly describe $s_{3}$.

For in order for $s_{3}$ to be fittingly described by 'Sam is sad or (Sam is bad and Sam is ill)', one of the following conditions should hold:

(i) $s_{3}$ is fittingly described by $\varnothing \mid$ 'Sam is sad';

(ii) $s_{3}$ is fittingly described by $\varnothing \mid$ 'Sam is bad', 'Sam is ill';

(iii) $s_{3}$ is fittingly described by $\varnothing \mid$ 'Sam is sad', 'Sam is bad', 'Sam is ill'.

But (i) fails because the supersentence leaves aside a part of $s_{3}$, namely $s_{2}$; (ii) fails because the supersentence leaves aside a part of $s_{3}$, namely $s_{1}$, and also because part of the supersentence, namely 'Sam is bad', does not describe any aspect of the situation; and (iii) fails because, and in the previous case, 'Sam is bad' describes no aspect of the situation.

Similar considerations hold in the sentential framework. Situation $s_{3}$ exactly verifies '(Sam is sad or Sam is bad) and (Sam is sad or Sam is ill)', and in order for it to also exactly verify 'Sam is sad or (Sam is bad and Sam is ill)', one of the following conditions should hold:

(i) $s_{3}$ exactly verifies 'Sam is sad';

(ii) $s_{3}$ is the fusion of two situations $t$ and $u$ such that $t$ exactly verifies 'Sam is bad' and $u$ 'Sam is ill';

(iii) $s_{3}$ is the fusion of three situations $t, u$ and $v$ such that $t$ exactly verifies 'Sam is sad', $u$ 'Sam is bad' and $v$ 'Sam is ill'.

Yet this is not the case: (i) fails because a part of $s_{3}$, namely $s_{2}$, is not relevant to the truth of 'Sam is sad'; (ii) fails because no part of $s_{3}$ verifies 'Sam is bad'; and (iii) fails for the same reason.

Fine's (forthcoming) state space semantics for Angell's system differs from the sentential semantics introduced above in just two respects: (i) it assumes that every atom of the language has falsifiers and verifiers in every model, and (ii) it takes $A \approx B$ to hold in a model iff the convex closure of the set of $A$ 's verifiers is identical to the convex closure of the set of $B$ 's verifiers, where the convex closure of the set of verifiers of a sentence $A$ is the set of all situations $s$ such that for some situations $t$ and $u$ such that $t \sqcup s=s$ and $s \bigsqcup u=u$, both $t$ and $u$ verify $A$. Given these modifications, we can readily see that A11 holds in every model (see Fine's theorem 14).

§4. Definitions and facts. We shall say that two sentences $A$ and $B$ are provably equivalent-in symbols: $A \equiv B$-when $A \approx B$ is a theorem of the system (given R1, $\mathrm{R} 2$ and the fact that $A \approx A$ is a theorem, provable equivalence is indeed an equivalence relation). 
Lemma 4.1 (Positive Substitution). Let $A$ and $B$ be sentences such that $A$ has occurrences in $B$ which are not in the scope of $\neg$. Let then $B^{\prime}$ result from $B$ by replacing some such occurrences of $A$ by a sentence $A^{\prime}$ that is provably equivalent to $A$. Then $B^{\prime}$ and $B$ are provably equivalent.

Proof. By induction on the complexity of $B$. If $B$ is atomic, the conclusion is immediate. Assume that the result holds up to a certain degree of complexity, and consider $B$ with the next degree. Then $B$ must be either of the form $C \wedge D$ or of the form $C \vee D$. In order to get the result, one can use the following derived rules of the system:

- $A \approx B, C \approx D / A \wedge C \approx B \wedge D$

- $A \approx B, C \approx D / A \vee C \approx B \vee D$

The first rule derives from A3, R2 and R3, and the second one from A6, R2 and R4.

We define a conjunction from a given nonempty set of sentences as any sentence that can be built using all and only the members of the set via the formation rule for conjunction; a sentence in the set may occur any number of times in the resulting conjunction, and in case the set comprises only one sentence, the sentence counts as a conjunction from the set. We define a disjunction from a nonempty set of sentences in the same way, appealing to the formation rule for disjunction rather than to the rule for conjunction. Using A2-A4 and Positive Substitution, one can show that any two conjunctions from the same set of sentences are provably equivalent, and using A5-A7 and Positive Substitution, one can show that the same holds for disjunctions.

Let a conjunctive form be a conjunction from a nonempty set of literals, and a disjunctive normal form a disjunction from a nonempty set of conjunctive forms. The components of a disjunctive normal form are the elements of the set from which it is generated. We associate to each finite nonempty set of literals $s$ a given conjunction $\Lambda s$ from it, and to each conjunctive form $A$ the set $\operatorname{lit}(A)$ of all the literals that compose it. (Here and below I use $s, t, u$ and variants thereof for sets of literals, anticipating that such sets will play the role of situations in the completeness proofs.) By a previous remark, every conjunctive form $A$ is provably equivalent to every conjunction from $\operatorname{lit}(A)$, and so in particular to $\bigwedge \operatorname{lit}(A)$. Conversely, if two conjunctive forms $A$ and $B$ are provably equivalent, then $\operatorname{lit}(A)=\operatorname{lit}(B)$.

LEMMA 4.2 (Disjunctive normal forms). Every sentence is provably equivalent to a disjunctive normal form.

Proof. A standard proof can be given using A1, A8, A9, A10, R1, R2 and Positive Substitution.

We associate to each sentence $A$ a specific disjunctive normal form $\hat{A}$ that is provably equivalent to it.

Let us say that $A$ is disjunctively contained in $B$-in symbols: $A \leq B$-iff $B \equiv A \vee B$ (i.e. iff $B \approx A \vee B$ is a theorem). Disjunctive containment will play a central role in the completeness proof. Here are some of its important properties:

LEMMA 4.3 (Properties of disjunctive containment).

1. $A \equiv B$ iff both $A \leq B$ and $B \leq A$.

2. If $A \leq B, A \equiv A^{\prime}$ and $B \equiv B^{\prime}$, then $A^{\prime} \leq B^{\prime}$.

3. $\leq$ is reflexive and transitive.

4. If $A \leq B$, then $A \wedge C \leq B \wedge C$. 
5. If $A \leq B$ and $A^{\prime} \leq B^{\prime}$, then $A \wedge A^{\prime} \leq B \wedge B^{\prime}$.

6. If $A_{1} \leq B_{1}, \ldots, A_{n} \leq B_{n}(n \geq 1)$, and if $C$ is a conjunction from the set of the $A_{i} s$ and $D$ a conjunction from the set of the $B_{i}$ s, then $C \leq D$.

7. If $A \leq B$, then $A \vee C \leq B \vee C$.

8. If $A \leq B$ and $A^{\prime} \leq B^{\prime}$, then $A \vee A^{\prime} \leq B \vee B^{\prime}$.

9. If $A_{1} \leq B_{1}, \ldots, A_{n} \leq B_{n}(n \geq 1)$, and if $C$ is a disjunction from the set of the $A_{i} s$ and $D$ a disjunction from the set of the $B_{i} s$, then $C \leq D$.

10. $A \wedge B \leq A \vee B$.

11. If $C$ is a conjunction from as set of sentences and $D$ a disjunction from the very same set, then $C \leq D$.

Proof.

1. Left to right: by A5, R1, R2 and R4. Right to left: by A6, R1 and R2.

2. By Positive Substitution.

3. Reflexivity is immediate by A5. For transitivity, suppose that (i) $A \leq B$, i.e. $B \equiv$ $A \vee B$ and (ii) $B \leq C$, i.e. $C \equiv B \vee C$. By (i) and R4, $B \vee C \equiv(A \vee B) \vee C$, and then by $\mathrm{A} 7, B \vee C \equiv A \vee(B \vee C)$. By (ii) and Positive Substitution, then, $C \equiv A \vee C$, i.e. $A \leq C$.

4. Suppose $A \leq B$, i.e. $B \equiv A \vee B$. By R3, then, $B \wedge C \equiv(A \vee B) \wedge C$, and so by A3, $B \wedge C \equiv C \wedge(A \vee B)$. By A10, it follows that $B \wedge C \equiv(C \wedge A) \vee(C \wedge B)$. By A3 and Positive Substitution, we finally conclude that $B \wedge C \equiv(A \wedge C) \vee(B \wedge C)$, i.e. $A \wedge C \leq B \wedge C$.

5. By A 3 and points 2 and 4 of this lemma.

6. The case $n=1$ follows from point 2 of this lemma. Suppose the result holds all $k$ with $1 \leq k \leq n$ and suppose that $A_{1} \leq B_{1}, \ldots, A_{n+1} \leq B_{n+1}$. Let then $C$ be a conjunction from $\left\{A_{1}, \ldots, A_{n+1}\right\}, D$ a conjunction from the same set, $C^{\prime}$ a conjunction from $\left\{A_{1}, \ldots, A_{n}\right\}$, and $D^{\prime}$ a conjunction from the same set. Then $C \equiv$ $C^{\prime} \wedge A_{n+1}, D \equiv D^{\prime} \wedge A_{n+1}$, and by induction hypothesis $C^{\prime} \leq D^{\prime}$. By R3 and point 2 of this lemma, then, $C \leq D$.

7. Suppose $A \leq B$, i.e. $B \equiv A \vee B$. By R4, then, $B \vee C \equiv(A \vee B) \vee C$, and so by A5 and Positive Substitution, $B \vee C \equiv(A \vee B) \vee(C \vee C)$. By A6, A7 and Positive Substitution, it follows that $B \vee C \equiv(A \vee C) \vee(B \vee C)$, i.e. $A \vee C \leq B \vee C$.

8. By A6 and points 2 and 7 of this lemma.

9. The case $n=1$ follows from point 2 of this lemma. Suppose the result holds all $k$ with $1 \leq k \leq n$ and suppose that $A_{1} \leq B_{1}, \ldots, A_{n+1} \leq B_{n+1}$. Let then $C$ be a disjunction from $\left\{A_{1}, \ldots, A_{n+1}\right\}, D$ a disjunction from the same set, $C^{\prime}$ a disjunction from $\left\{A_{1}, \ldots, A_{n}\right\}$, and $D^{\prime}$ a disjunction from the same set. Then $C \equiv C^{\prime} \vee A_{n+1}$, $D \equiv D^{\prime} \vee A_{n+1}$, and by induction hypothesis $C^{\prime} \leq D^{\prime}$. By R4 and point 2 of this lemma, then, $C \leq D$.

10. Consider the following sentences:

1. $(A \wedge B) \vee(A \vee B)$

2. $((A \wedge B) \vee(B \wedge A)) \vee(A \vee B)$

3. $((A \wedge B) \vee A) \vee((B \wedge A) \vee B)$

4. $((A \wedge B) \vee(A \wedge A)) \vee((B \wedge A) \vee(B \wedge B))$

5. $(A \wedge(B \vee A)) \vee(B \wedge(A \vee B))$ 
6. $(A \wedge(B \vee A)) \vee(B \wedge(B \vee A))$

7. $((B \vee A) \wedge A) \vee((B \vee A) \wedge B)$

8. $(B \vee A) \wedge(A \vee B)$

9. $A \vee B$

Each of $1-8$ is provably equivalent to the next sentence:

$1 \equiv 2$ by A3, A5 and Positive Substitution.

$2 \equiv 3$ by A6, A7 and Positive Substitution.

$3 \equiv 4$ by $\mathrm{A} 2$ and Positive Substitution.

$4 \equiv 5$ by A10 and Positive Substitution.

$5 \equiv 6$ by $\mathrm{A} 6$ and Positive Substitution.

$6 \equiv 7$ by $\mathrm{A} 3$ and Positive Substitution.

$7 \equiv 8$ by $\mathrm{A} 10$.

$8 \equiv 9$ by $\mathrm{A} 2$, A6 and Positive Substitution.

Hence $A \vee B \equiv(A \wedge B) \vee(A \vee B)$, i.e. $A \wedge B \leq A \vee B$.

11. By induction of the size of the set of sentences. If the set comprises just one element $A$, then both $C \equiv A$ and $D \equiv A$, and so $C \equiv D$, and so, by the reflexivity of $\leq$ and point 2 of this lemma, $C \leq D$. Suppose the result holds for the case of sets of $k$ sentences from $k=1$ to a given $n \geq 1$, and consider $C$ a conjunction from a set of $n+1$ sentences $A_{1}, \ldots, A_{n+1}$ and $D$ a disjunction from the same set. Let then $C^{\prime}$ be a conjunction from $\left\{A_{1}, \ldots, A_{n}\right\}$ and $D^{\prime}$ a disjunction from the same set. Then $C \equiv C^{\prime} \wedge A_{n+1}$ and $D \equiv D^{\prime} \vee A_{n+1}$. By induction hypothesis, we also have $C^{\prime} \leq D^{\prime}$, and so by point 4 of this lemma, $C^{\prime} \wedge A_{n+1} \leq D^{\prime} \wedge A_{n+1}$, and so by point 2 of this lemma, $C \leq D^{\prime} \wedge A_{n+1}$. By point 10 of this lemma, $D^{\prime} \wedge A_{n+1} \leq D^{\prime} \vee A_{n+1}$, and so by point 2 of this lemma $D^{\prime} \wedge A_{n+1} \leq D$. By the transitivity of $\leq$, it follows that $C \leq D$.

This lemma allows one to prove the following fact, which will be central to the completeness proofs to come:

LEMMA 4.4 (Sufficient condition for disjunctive containment). Let $A$ and $B$ be two disjunctive normal forms, and suppose that for every component $A^{\prime}$ of $A$, there is a covering $\left(s_{i}\right)_{i \in I}$ of lit $\left(A^{\prime}\right)$ such that for all $i \in I$, there is a component $B^{\prime}$ of $B$ such that $s_{i}=\operatorname{lit}\left(B^{\prime}\right)$. Then $A \leq B$.

Proof. Let $A$ and $B$ be as stated. Let $\left(A_{j}\right)_{j \in J}$ be the components of $A$, and consider an arbitrary $A_{j}$. By hypothesis, there is a covering $\left(s_{i}\right)_{i \in I}$ of $\operatorname{lit}\left(A_{j}\right)$ such that for all $i \in I$, there is a component $B^{\prime}$ of $B$ such that $s_{i}=\operatorname{lit}\left(B^{\prime}\right)$, and hence such that $\bigwedge s_{i} \equiv B^{\prime}$. Select, for each $i \in I$ a given component $B_{i}$ satisfying this condition. Let then $B^{\wedge}$ be a conjunction from the set of the $B_{i}$ s and $B^{\vee}$ be a disjunction from the same set. Clearly $B^{\vee} \leq B$, and by point 11 of the previous lemma and the transitivity of $\leq$, it follows that $B^{\wedge} \leq B$. Given that for all $i \in I, \bigwedge s_{i} \equiv B_{i}$, by point 6 of the previous lemma we have $A_{j} \equiv B^{\wedge}$. By point 2 of the previous lemma, it follows that $A_{j} \leq B$. This holds for any $j \in J$, and so by point 9 of the previous lemma we have $A \leq B$.

§5. Soundness and completeness. Let us start with the supersentential semantics.

THEOREM 5.1 (Soundness for the supersentential semantics). Every theorem of the system is supervalid. 
Proof. It is almost immediate that the axioms of the system are supervalid, and that Rules 1 and 2 preserve supervalidity. Things are not straightforward in the case of Rules 3 and 4, but a short detour will help. Let us define another notion of validity as follows:

$A \approx B$ is supervalid* iff for all models $M$ and all situations $s$ in $M$ : for all finite sets of sentences $R, s \Vdash \varnothing \mid R, A$ iff $s \Vdash \varnothing \mid R, B$.

Clearly, supervalidity* is at least as strong as supervalidity, and so by establishing that every theorem of the system is supervalid* one will establish soundness. It is fairly easy to prove that the axioms of the system are supervalid*, and that the rules preserve supervalidity* (including Rules 3 and 4). Thus, every theorem of the system is supervalid*, and hence supervalid.

Notice that given the completeness result below, supervalidity* as defined in the proof above and supervalidity are coextensive.

In order to establish completeness, we define the supermodel $M=\langle S, \Vdash\rangle$ where $S$ is the set of all finite nonempty sets of literals, and for every basic supersentence $L|R, s \Vdash L| R$ iff $L$ is the set of all negated atoms in $s$ and $R$ the set of all nonnegated atoms in $s$.

LEMMA 5.2. Let $s$ be a situation of $M$ and $\left(A_{1}, \ldots, A_{n}\right)(n \geq 1)$ a family of conjunctive forms. Then: $s \Vdash \varnothing \mid A_{1}, \ldots, A_{n}$ iff $s=\bigcup_{1 \leq i \leq n} \operatorname{lit}\left(A_{i}\right)$.

Proof. By semantic Clauses 2 and 3 and the definition of $\Vdash$.

THEOREM 5.3 (Completeness for the supersentential semantics). Every supervalid equivalence is a theorem.

Proof. Suppose that $A \approx B$ is not a theorem. Then by Lemma 4.3(1), either $A \not \leq B$ or $B \not \leq A$. Suppose $A \not \leq B$ (the other case is exactly similar). Then by Lemma 4.3(2),

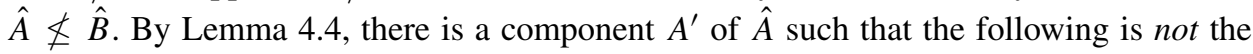
case:

$(\sharp)$ There is a covering $\left(s_{i}\right)_{i \in I}$ of $\operatorname{lit}\left(A^{\prime}\right)$ such that for all $i \in I$, there is a component $B^{\prime}$ of $\hat{B}$ such that $s_{i}=\operatorname{lit}\left(B^{\prime}\right)$.

By Lemma $5.2, \operatorname{lit}\left(A^{\prime}\right) \vDash \varnothing \mid A^{\prime}$, and so by semantic Clause $5, \operatorname{lit}\left(A^{\prime}\right) \Vdash \varnothing \mid \hat{A}$, and hence by soundness $\operatorname{lit}\left(A^{\prime}\right) \Vdash \varnothing \mid A$. Suppose, for reductio, that $\operatorname{lit}\left(A^{\prime}\right) \Vdash \varnothing \mid B$. Then by soundness, $\operatorname{lit}\left(A^{\prime}\right) \Vdash \varnothing \mid \hat{B}$. By semantic Clause 5 , there should be a family $\left(B_{1}^{\prime}, \ldots, B_{n}^{\prime}\right)$ $(n \geq 1)$ of components of $\hat{B}$ such that $\operatorname{lit}\left(A^{\prime}\right) \Vdash \varnothing \mid B_{1}^{\prime}, \ldots, B_{n}^{\prime}$. But by Lemma 5.2, this requires that $(\sharp)$ be true. So, $\operatorname{lit}\left(A^{\prime}\right) \nVdash \varnothing \mid B$. Hence, $A \approx B$ does not hold in $M$ and is therefore not supervalid.

Let us now turn to the sentential semantics. Soundness is straightforward. In order to establish completeness, let us follow Fine (forthcoming) and define a model $N=$ $\langle S, \bigcup, F, V\rangle$ where $S$ is the set of all sets of literals, $\bigcup$ is set-theoretic union, and for each atom $p, V(p)=\{\{p\}\}$ and $F(p)=\{\{\neg p\}\}$.

LEMmA 5.4. Let $s$ be a situation of $N$ and $A$ a conjunctive form. Then: $s \vDash A$ iff $s=\operatorname{lit}(A)$.

Proof. By induction on the number of literals occurring in $A$. If $A$ is a literal, then the conclusion follows from the definitions of $F$ and $V$. Let then $A$ be a conjunctive form $B \wedge C$. Then $s \vDash B \wedge C$ iff for some situations $t$ and $u$ of $N$ such that $s=t \bigcup u, t \vDash B$ and 
$u \vDash C$ (by the verification clause for conjunction), iff for some situations $t$ and $u$ of $N$ such that $s=t \bigcup u, t=\operatorname{lit}(B)$ and $u=\operatorname{lit}(C)$ (by induction hypothesis), iff $s=\operatorname{lit}(B \wedge C)$.

THEOREM 5.5 (Completeness for the sentential semantics). Every valid equivalence is a theorem.

Proof. The proof is almost exactly like the previous one. Suppose that $A \approx B$ is not a theorem. Then by Lemma 4.3(1), either $A \not \leq B$ or $B \not \leq A$. Suppose for illustration that $A \not \leq B$. Then by Lemma 4.3(2), $\hat{A} \not \leq \hat{B}$. By Lemma 4.4, there is a component $A^{\prime}$ of $\hat{A}$ such that $(\sharp)$ from the previous proof is not the case. By Lemma $5.4, \operatorname{lit}\left(A^{\prime}\right) \vDash A^{\prime}$, and so by the verification clause for $\operatorname{disjunction}, \operatorname{lit}\left(A^{\prime}\right) \vDash \hat{A}$, and hence by soundness $\operatorname{lit}\left(A^{\prime}\right) \vDash A$. Suppose, for reductio, that $\operatorname{lit}\left(A^{\prime}\right) \vDash B$. Then by soundness, $\operatorname{lit}\left(A^{\prime}\right) \vDash \hat{B}$. By the verification clause for disjunction, there should be a family $\left(B_{1}^{\prime}, \ldots, B_{n}^{\prime}\right)(n \geq 1)$ of components of $\hat{B}$ and a covering $\left(s_{1}, \ldots, s_{n}\right)$ of $\operatorname{lit}\left(A^{\prime}\right)$ such that $s_{i} \vDash B_{i}^{\prime}$ for all $i$. But by Lemma 5.4, this requires that $(\sharp)$ be true. So, $\operatorname{lit}\left(A^{\prime}\right) \not \models B$. Hence, $A \approx B$ does not hold in $N$ and is therefore not valid.

The previous results yield straightforward semantic characterisations of the system resulting from our system for factual equivalence by substituting A11 for A10-a system we shall call the dual system.

By a straightforward induction on the length of the proofs, one can establish the following:

LEMMA 5.6. For all sentences $A$ and $B, A \approx B$ is a theorem of the dual system iff $\neg A \approx \neg B$ is a theorem of the original system, and $A \approx B$ is a theorem of the original system iff $\neg A \approx \neg B$ is a theorem of the dual system.

We thus have:

THEOREM 5.7 (Soundness and completeness for the dual system). $A \approx B$ is a theorem of the dual system iff $\neg A \approx \neg B$ is supervalid, iff $\neg A \approx \neg B$ is valid.

Let $A^{\circ}$ be the result of replacing in $A$ every occurrence of $\wedge$ by $\vee$ and every occurrence of $\vee$ by $\wedge$. We also have:

LEMMA 5.8. For all sentences $A$ and $B, A \approx B$ is a theorem of the dual system iff $A^{\circ} \approx B^{\circ}$ is a theorem of the original system, and $A \approx B$ is a theorem of the original system iff $A^{\circ} \approx B^{\circ}$ is a theorem of the dual system.

And so:

THEOREM 5.9 (Soundness and completeness for the dual system). $A \approx B$ is a theorem of the dual system iff $A^{\circ} \approx B^{\circ}$ is supervalid, iff $A^{\circ} \approx B^{\circ}$ is valid.

It would be interesting to identify a Hilbert-style system whose theorems are just the sentences $A \approx B$ such that both $A \approx B$ and $\neg A \approx \neg B$ are supervalid (or equivalently: valid).

§6. Factual equivalence on the fitting-or-truncated conception of description. We have so far worked with a conception of descriptions as fitting. What logic of factual equivalent do we get if we work instead with a conception of descriptions as fitting-ortruncated?

On the sentential approach, the semantic clauses for negation stay the same, but the clauses for conjunction and disjunction become the following familiar-sounding clauses: 
- $\quad s$ verifies $A \wedge B$ iff $s$ verifies $A$ and $s$ verifies $B$;

- $s$ falsifies $A \wedge B$ iff $s$ falsifies $A$ or $s$ falsifies $B$

- $s$ verifies $A \vee B$ iff $s$ verifies $A$ and $s$ verifies $B$;

- $s$ falsifies $A \vee B$ iff $s$ falsifies $A$ or $s$ falsifies $B$.

An immediate consequence is that the distributivity axiom A11, i.e. $A \vee(B \wedge C) \approx$ $(A \vee B) \wedge(A \vee C)$, is valid on the fitting-or-truncated description approach. We see here in a particularly vivid way the import of the distinction between the tight notion of a fitting description and the loose notion of a fitting-or-truncated description.

Due to the shape of the semantic clauses above, the operation of fusion in models no longer plays a role in the semantics, and so it can simply be ignored. Say that $A \Rightarrow B$ iff relative to any model, every situation that verifies $A$ also verifies $B$ (thus, $A \approx B$ is valid iff both $A \Rightarrow B$ and $B \Rightarrow A$ ). Then $A \Rightarrow B$ iff $B$ is a first-degree entailment of $A$ in the sense of Anderson \& Belnap $(1962,1963)$. This is fairly easy to prove if we use the following semantical characterisation of first-degree entailment (see e.g. Priest, 2008, pp. 143-144). Define a valuation as a binary relation between atomic sentences and the truth-values $\mathrm{T}$ and $\mathrm{F}$. Truth $(\models)$ and falsity $(\Rightarrow)$ relative to a valuation $v$ are defined via the following clauses:

- For $A$ atomic: $v \vDash A$ iff $v(A, \mathrm{~T})$;

- For $A$ atomic: $v \neq A$ iff $v(A, \mathrm{~F})$;

- $v \vDash \neg A$ iff $v \neq A$;

- $v \exists \neg A$ iff $v \vDash A$;

- $v \vDash A \wedge B$ iff $v \vDash A$ and $v \vDash B$;

- $v \Rightarrow A \wedge B$ iff $v \neq A$ or $v \neq B$

- $v \vDash A \vee B$ iff $v \vDash A$ or $v \vDash B$;

- $v \Rightarrow A \vee B$ iff $v \Rightarrow A$ and $v=B$.

Then $B$ is a first-degree entailment of $A$ iff for every valuation $v$, if $v \vDash A$, then $v \vDash B$.

The logic of factual equivalence one gets on the fitting-or-truncated conception of description is thus the logic of mutual first-degree entailment: $A \approx B$ is valid iff $A$ and $B$ are first-degree entailments of each other. ${ }^{8}$ On the proof-theoretic side, an adequate system is obtained by adding two axioms to our original system for factual equivalence, namely

$$
\text { A11. } A \vee(B \wedge C) \approx(A \vee B) \wedge(A \vee C)
$$

and

$$
\text { A12. } A \approx A \vee(A \wedge B),
$$

or, equivalently given postulates of the original system,

$$
\text { A13. } A \approx A \wedge(A \vee B) \text {. }
$$

For as we saw, adding A11 to the original system yields Angell's logic of analytic equivalence, and adding A12 or A13 to Angell's logic yields the logic of mutual first-degree entailment (Angell, 1977).

On the supersentential approach, moving from the fitting to the fitting-or-truncated conception makes description by supersentences collapse into descriptions by the component sentences-more precisely, $L \mid R$ describes situation $s$ iff $s$ is described by each $A \mid \varnothing$

8 Fine (forthcoming) establishes, in effect, this result. 
with $A \in L$ and by each $\varnothing \mid A$ with $A \in R$. As a result, the logic of factual equivalence generated by the supersentential semantics is again the logic of mutual first-degree entailment.

\$7. Factual equivalence and grounding. The conception of factual equivalence resulting from the fitting-or-truncated description semantics certainly has its virtues, but it is ill-adapted for certain theoretical endeavours. Here I wish to focus on the topic I deal with in "Grounding and Truth-Functions", namely the theory of grounding. 9

According to view put forward in that paper, grounding and factual equivalence are connected through substitution principles, like the following:

- If $r$ and $r^{\prime}$ are factually equivalent and $\ulcorner$ the facts that $p$, that $q, \ldots$ (together) ground the fact that $r\urcorner$ is true, then so is $\ulcorner$ the facts that $p$, that $q, \ldots$ (together) ground the fact that $\left.r^{\prime}\right\urcorner$.

If this principle is taken for granted, then it is clear that the logic of mutual first-degree entailment cannot be the correct logic of factual equivalence. In fact, axioms A11, A12 and A13 yield trouble.

Let us first go back to sentences that were used in Section 3 to argue against A11, and let us suppose that both 'Sam is sad' and 'Sam is ill' are true. It is then plausible to hold that

(a) The fact that Sam is sad grounds the fact that (Sam is sad or Sam is bad) and

(b) The fact that Sam is ill grounds the fact that (Sam is sad or Sam is ill).

Then plausibly,

(c) The facts that Sam is sad and that Sam is ill ground the fact that ((Sam is sad or Sam is bad) and (Sam is sad or Sam is ill)).

Then by A11 and the substitution principle,

(d) The facts that Sam is sad and that Sam is ill ground the fact that (Sam is sad or (Sam is bad and Sam is ill)).

But, intuitively, (d) is false. For we may suppose that Sam is not bad, in which case 'Sam is bad and Sam is ill' will false, and accordingly the fact that Sam ill will play no role whatsoever in grounding the disjunctive fact. (The fact that Sam is sad will do the work.) We actually do not need to suppose that Sam is not bad to make the point. For suppose that Sam is bad. Then the fact that Sam is ill does help ground the disjunctive fact: the latter is grounded in the former in combination with the fact that Sam is bad. But clearly, (d) fails: the disjunctive fact is not grounded in the fact that Sam is ill in combination with the fact that Sam is sad. ${ }^{10}$

Against A12, one can reason as follows. Granted that Brutus is a dog, it is plausible to hold that

9 I am here assuming that the reader is familiar with the notion of grounding. For useful surveys, see Correia \& Schneider (2012), Trogdon (2013), and Bliss \& Trogdon (2014). Importantly, in "Grounding and Truth-Functions" I focus on a worldly conception of grounding. On the worldly / conceptual distinction, see Correia (2010) and Correia \& Schnieder (2012, sec. 3.3).

10 I should mention that Krämer \& Roski (2015) have independently made the very same objection to the combination of A11 and the substitution principle for grounding. 
(e) The facts that Brutus is a dog and that $2+2=4$ ground the fact that (Brutus is a dog and $2+2=4$ ).

Then plausibly,

(f) The facts that Brutus is a dog and that $2+2=4$ ground the fact that (Brutus is a dog or (Brutus is a dog and $2+2=4)$ ).

Then by A12 and the substitution principle,

(g) The facts that Brutus is a dog and that $2+2=4$ ground the fact that Brutus is a dog.

But this conclusion is objectionable for two reasons: first, the fact that Brutus is a dog surely does not help ground itself $;{ }^{11}$ and second, the fact that $2+2=4$ surely does not help ground the fact that Brutus is a dog.

Notice that since A12 is entailed by A13 in the presence of the postulates of the original system, if we suppose the latter given, then the previous objection to A12 counts as an objection to A13. But since the postulates of the system which secure that A13 entails A12 might be taken to be objectionable, it is safer to argue directly against A13. So, suppose that

(h) The fact that Brutus is a dog grounds the fact that Brutus is an animal and that

(i) The fact that $2+2=4$ grounds the fact that (Brutus is an animal or $2+2=4$ ).

Then presumably,

(j) The facts that Brutus is a dog and that $2+2=4$ ground the fact that (Brutus is an animal and (Brutus is an animal or $2+2=4)$ ).

Then by A13 and the substitution principle,

(k) The facts that Brutus is a dog and that $2+2=4$ ground the fact that Brutus is an animal.

But the fact that $2+2=4$ certainly does not help ground the fact that Brutus is an animal.

Thus, if the proposed substitution principle for grounding is to be accepted, then each of A11, A12 and A13 must be rejected. As previously mentioned, in the 2010 paper I advocate the view that the logic of factual equivalence is Angell's logic of analytic equivalence. A12 and A13 are not theorems of the latter, but A11 is. I suggest that the logic of the notion of factual equivalence that is relevant for the theory of grounding is the logic validated by the fitting description semantics.

§8. Acknowledgments. I am grateful to Stephan Krämer and two referees of this journal for helpful comments on drafts of this paper. This work was carried out while I was in charge of the Swiss National Science Foundation projects 'Grounding - Metaphysics, Science, and Logic' (Neuchâtel, CRSII1-147685) and 'The Nature of Existence: Neglected Questions at the Foundations of Ontology' (Neuchâtel, 100012-150289), and a member of the Spanish Ministry of Economy and Competitiveness 'The Makings of Truth: Nature, Extent, and Applications of Truthmaking' (Barcelona, FFI2012-35026).

11 Grounding orthodoxy has it that grounding is irreflexive, i.e. that no fact can help ground itself. I am not relying on such a strong claim here. See Bliss \& Trogdon (2014) on putative violations of irreflexivity. 


\section{BIBLIOGRAPHY}

Anderson, A. R., \& Belnap, N. D. (1962). Tautological entailments. Philosophical Studies, 13, 9-24.

Anderson, A. R., \& Belnap, N. D. (1963). First-degree entailments. Mathematische Annalen, 149, 302-319.

Angell, R. B. (1977). Three systems of first degree entailment. Journal of Symbolic Logic, 42, 147.

Angell, R. B. (1989). Deducibility, entailment and analytic containment. In Norman, J. and Sylvan, R., editors. Directions in Relevant Logic. Dordrecht: Kluwer Academic Publishers, pp. 119-143.

Barker, S., \& Jago, M. (2012). Being positive about negative facts. Philosophy and Phenomenological Research, 85(1), 117-138.

Bliss, R., \& Trogdon, K. (2014). Metaphysical grounding. In Zalta, E. N., editor. The Stanford Encyclopedia of Philosophy (Winter 2014 edition) http://plato.stanford .edu/archives/win2014/entries/grounding/.

Cobreros, P., Égré, P., Ripley, D., \& van Rooij, R. (2014). Pragmatic interpretations of vague expressions: Strongest meaning and nonmonotonic consequence. Journal of Philosophical Logic. To appear.

Correia, F. (2004). Semantics for analytic containment. Studia Logica, 77, 87-104.

Correia, F. (2010). Grounding and truth-functions. Logique \& Analyse, 53(211), 251-279.

Correia, F., \& Schnieder, B. (2012). Grounding: An opinionated introduction. In Correia, F. and Schneider, B., editors. Metaphysical Grounding: Understanding the Structure of Reality. Cambridge: Cambridge University Press, pp. 1-36.

Ferguson, T. M. (2014). Faulty Belnap computers and subsystems of FDE. Journal of Logic and Computation. To appear.

Fine, K. (2012a). Guide to ground. In Correia, F. and Schneider, B., editors. Metaphysical Grounding: Understanding the Structure of Reality. Cambridge: Cambridge University Press, pp. 37-80.

Fine, K. (2012b). The pure logic of ground. Review of Symbolic Logic, 25(1), 1-25.

Fine, K. (2014). Truthmaker semantics for intuitionistic logic. Journal of Philosophical Logic, 43(2), 549-77.

Fine, K. (forthcoming). Angellic content.

Fine, K. (ms a). A theory of truth-conditional content I.

Fine, K. (ms b). A theory of truth-conditional content II.

Fine, K. (ms c). Truthmaker semantics.

Jennings, R. E., \& Chen, Y. (2013). FDE: A logic of clutters. In Tanaka, K., Berto, F., Mares, E., and F. Paoli, editors. Paraconsistency: Logic and Applications. New York: Springer, pp. 163-172.

Krämer, S., \& Roski, S. (2015). A note on the logic of worldly ground. Thought: A Journal of Philosophy, 4(1), 59-68.

Priest, G. (2008). An Introduction to Non-Classical Logic: From If to Is. New York: Cambridge University Press.

Trogdon, K. (2013). An introduction to grounding. In Hoeltje, M., Schnieder, B., and Steinberg, A., editors. Varieties of Dependence. Basic Philosophical Concepts. Munich: Philosophia Verlag, pp. 97-122.

Van Fraassen, B. (1969). Facts and tautological entailments. Journal of Philosophy, 66, pp. 477-487. 


\section{INSTITUT DE PHILOSOPHIE \\ UNIVERSITÉ DE NEUCHÂTEL \\ ESPACE LOUIS-AGASSIZ 1 \\ 2000 NEUCHÂTEL \\ SWITZERLAND}

E-mail: fabrice.correia@unine.ch 